\title{
Qiliqiangxin Rescues Mouse Cardiac Function by Regulating AGTR1/TRPV1- Mediated Autophagy in STZ-Induced Diabetes Mellitus
}

\author{
Jing Tong Yan Lai Yi-An Yao Xue-Jun Wang Yu-Shuang Shi \\ Han-Jin Hou Jian-Yun Gu Fei Chen Xue-Bo Liu \\ Department of Cardiology, Shanghai Tongji Hospital, Tongji University, Shanghai, China
}

\section{Key Words}

Qiliqiangxin • AGTR1 • TRPV1 • Autophagy • Diabetes

\begin{abstract}
Background/Aims: To explore the potential role of qiliqiangxin (QLQX) A traditional Chinese medicine and the involvement of angiotensin II receptor type 1 (AGTR1) and transient receptor potential vanilloid 1 (TRPV1) in diabetic mouse cardiac function. Methods: Intragastric QLQX was administered for 5 weeks after streptozotocin (STZ) treatment. Additionally, Intraperitoneal injections of angiotensin II (Ang II) or intragastric losartan (Los) were administered to assess the activities of AGTR1 and TRPV1. Two-dimensional echocardiography and tissue histopathology were used to assess cardiac function Western blot was used to detect the autophagic biomarkers Such as light chain 3 P62 and lysosomal-associated membrane protein 2 And transmission electron microscopy was used to count the number of autophagosomes. Results: Decreased expression of TRPV1 and autophagic hallmarks and reduced numbers of autophagolysosomes as well as increased expression of angiotensin converting enzyme 1 and AGTR1 were observed in diabetic hearts. Blocking AGTR1 with Los mimicked the QLQXmediated improvements in cardiac function Alleviated myocardial fibrosis and enabled autophagy Whereas Ang II abolished the beneficial effects of QLQX in wild type diabetic mice but not in TRPV1 ${ }^{--}$diabetic mice. Conclusions: QLQX may improve diabetic cardiac function by regulating AGTR1/ TRPV1-mediated autophagy in STZ-induced diabetic mice.
\end{abstract}

\section{Introduction}

Cardiovascular diseases, including myocardial ischemia and cardiomyopathy, are the most common complications among diabetic patients [1]. Current solutions aimed at

T. Jing and L. Yan contributed equally to this work.

Chen Fei

and Liu Xue-Bo

KARGER
Department of Cardiology, Shanghai Tongji Hospital, Tongji University 389 Xincun Road, Shanghai 200065, Shanghai (China)

Tel. +862156051682, E-Mail liuxb70@hotmail.com, riverapt@126.com 


\section{Cellular Physiology Cell Physiol Biochem 2018;47:1365-1376

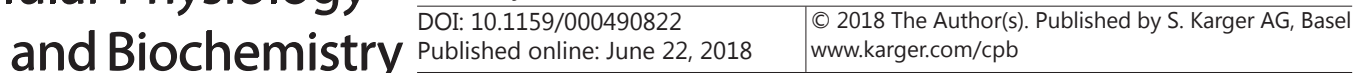 \\ Tong et al.: QLQX Improving Diabetic Cardiac Function}

improving diabetes and preventing further cardiovascular complications include blood sugar maintenance, lifestyle changes, and pharmacological interventions [2]. However, the pathogenesis of cardiovascular complications and the optimum treatments for diabetic patients remain unclear. Therefore, new therapeutic interventions to reduce cardiovascular complications are needed.

Qiliqiangxin (QLQX), a traditional Chinese herb, has been shown to be an effective and safe treatment for chronic heart failure (CHF) and significantly alleviates N-terminal pro brain natriuretic peptide (NT-pro-BNP) levels in CHF patients [3] by preventing myocardial remodeling [4, 5], blocking $\mathrm{Ca}^{2+}$ channels [6], regulating autophagy [7], ameliorating cardiomyocyte metabolism [8], and increasing cardiac contractility [4, 8]. However, it is not known whether QLQX can improve cardiac dysfunction in diabetic patients. In our previous study, we found that QLQX exerts beneficial effects on endothelial function in diabetic rats by increasing serum nitric oxide (NO) levels, balancing the NO pathway, and inhibiting the expression of angiotensin converting enzyme 1 (ACE1) and angiotensin II receptor type 1 (AGTR1) [9]. It is believed that endothelial dysfunction and ACE1/AGTR1 could be associated with exacerbations of cardiac dysfunction and increase mortality rates in patients with CHF [10]. Therefore, we compared the effects of QLQX on cardiac dysfunction between diabetic and normal mice to explore whether QLQX confers significant beneficial effects.

The proposed mechanism for the pathogenesis of ACE1/AGTR1 in diabetic cardiomyopathy is highly complex. Recently, Issa reported that increased expression of transient receptor potential vanilloid 1 (TRPV1) protein is observed in rats with angiotensin II (Ang II)-dependent malignant hypertension, which indicates that TRPV1 may be an important contributing factor to these effects of Ang II [11]. Moreover, the role of the TRPV1 channel in the regulation of cardiovascular dysfunction in diabetes has been established [2]. Zheng demonstrated impaired TRPV1 activity and deficient levels of primary downstream transmitters, such as calcitonin gene related peptide (CGRP) and substance P (SP), in the hearts of diabetic mice and patients [12]. In addition, TRPV1 has been linked to a reduction in coronary blood flow and excessive production of reactive oxygen species (ROS) [13]. Therefore, we hypothesized that QLQX may exert protective effects via the regulation of AGTR1 and TRPV1 in diabetic hearts.

\section{Materials and Methods}

\section{Reagents}

Streptozotocin (STZ) and Ang II were purchased from Sigma-Aldrich (St Louis, MO, USA); monoclonal rabbit antibodies against adenosine monophosphate activated protein kinase (AMPK), p-APMK, p62, p-p62, beclin1, light chain 3 (LC3), lysosomal-associated membrane protein 2 (LAMP2), and $\alpha$-tubulin were obtained from Cell Signaling Technology (Denver, MA, USA); polyclonal rabbit antibodies against ACE-1, AGTR1, and TRPV1 were obtained from Epitomics Inc. (Burlingame, CA, USA); the Masson's trichrome staining kit was purchased from Beyotime (Haimen, China); and losartan (Los) was a kind gift from Merck \& Co., Inc. (Kenilworth, NJ, USA). QLQX compounds consisting of radix astragali, aconite root, ginseng, Salvia miltiorrhiza, Semen Lepidii Apetali, Cortex Periplocae Sepii Radicis, Rhizoma Alismatis, Carthamus Tinctorius, Polygonatum Odorati, seasoned orange peel, and Rumulus Cinnamomi, were kindly provided by Yiling Pharmaceutical Corporation (Shijiazhuang, China). The herbal drugs were authenticated and standardized with marker compounds according to the Chinese Pharmacopoeia 2005. The powdered drug was dissolved in normal saline.

\section{Animals}

The investigation was performed in accordance with the Guide for the Care and Use of Laboratory Animals published by the US National Institutes of Health (NIH revised 2011) and approved by the Institutional Animal Care and Use Committee of Tongji University. Eight-week-old C57BL/6J male mice were purchased from the Experimental Animal Centre, Chinese Academy of Sciences (Shanghai, China). TRPV1 deficient $(T R P V 1 \%)$ mice were a kind gift from Prof. Zheng of Zhejiang University. After $12 \mathrm{~h}$ of fasting, 


\section{Cellular Physiology Cell Physiol Biochem 2018;47:1365-1376 and Biochemistry Published \begin{tabular}{l|l} 
DOI: 10.1159/000490822 & $\begin{array}{l}\text { C } 2018 \text { The Author(s). Published by S. Karger AG, Basel } \\
\text { www.karger.com/cpb }\end{array}$
\end{tabular} \\ Tong et al.: QLQX Improving Diabetic Cardiac Function}

diabetes was induced by intraperitoneal injection of $150 \mathrm{mg} / \mathrm{kg}$ STZ. Mice were considered diabetic when glucose levels exceeded $16.4 \mathrm{mmol} / \mathrm{L}$ at $72 \mathrm{~h}$ after STZ injection. Control mice were injected with saline only.

\section{Drug intervention}

The QLQX dosage recommended by Yiling Pharmaceutical Corporation is $0.3-1.2 \mathrm{~g} \cdot \mathrm{kg}^{-1} \cdot \mathrm{d}^{-1}$. Since we

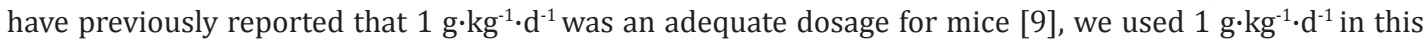
study. The dosage of Los was $50 \mathrm{mg} \cdot \mathrm{kg}^{-1} \cdot \mathrm{d}^{-1}$ as we reported previously [5], and the dosage of Ang II was 0.6 $\mathrm{mg} \cdot \mathrm{kg}^{-1} \cdot \mathrm{d}^{-1}$ as reported by Liu [14].

The mice were randomly divided into the following 8 groups: (1) untreated wild type (WT) mice as the normal control group; (2) untreated WT diabetic mice; (3) WT diabetic mice with intragastric QLQX; (4) WT diabetic mice with intragastric Los; (5) WT diabetic mice with intragastric QLQX and subcutaneous injections of Ang II; (6) untreated TRPV1\% diabetic mice; (7) TRPV1\% diabetic mice with intragastric QLQX; and (8) TRPV1\% diabetic mice with subcutaneous injections of Ang II. Untreated diabetic mice and normal control mice were administered with intragastric or subcutaneous injections of equal amounts of saline each day. Ang II or saline was administered by subcutaneous injections for 14 days as described previously [14]. The intragastric QLQX treatments continued for 5 weeks as per our previous report [9].

\section{Two-dimensional echocardiography}

Mice were anesthetized with pentobarbital sodium $(50 \mathrm{mg} / \mathrm{kg}$; Merck, Darmstadt, Germany) and subjected to transthoracic echocardiography to measure the left ventricular end-systolic internal diameter (LVESd), left ventricular end-diastolic internal diameter, and the ejection fraction (EF). The mice were then sacrificed by cervical dislocation.

\section{Histopathology}

Freshly dissected hearts were fixed in $10 \%$ (v/v) formalin for $24 \mathrm{~h}$, embedded in paraffin, sliced into 4-mm-thick sections, and stained with haematoxylin and eosin (H\&E) and Masson's trichrome to visualize cardiac hypertrophy and areas of interstitial and perivascular fibrosis by microscopy. The cardiac hypertrophic and fibrotic areas were measured using Image-Pro Plus 5 software (Media Cybernetics, Inc., MD).

\section{Transmission electron microscopy}

The freshly dissected hearts were fixed in 5\% glutaraldehyde and dehydrated in a graded series of ethanol solutions. After processing, the samples were observed using a transmission electron microscope.

\section{Western blot analysis}

Proteins were extracted from heart tissue with lysis buffer containing $50 \mathrm{mM}$ Tris (pH 7.4), $150 \mathrm{mM} \mathrm{NaCl}$, $1 \%$ Triton X-100, $1 \%$ sodium deoxycholate, $0.1 \%$ sodium dodecyl sulfate, $1 \mathrm{mM}$ ethylenediaminetetraacetic

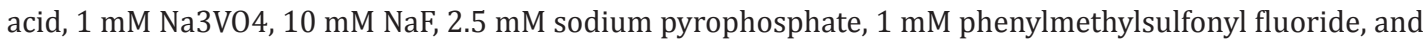
protease inhibitor cocktail (Cat. \# PIC02, Cytoskeleton), and equivalent amounts of protein were separated by sodium dodecyl sulfate-polyacrylamide gel electrophoresis and transferred to polyvinylidene fluoride membranes (Millipore, Billerica, MA). The membranes were blocked with 5\% non-fat dried milk for $2 \mathrm{~h}$. After incubation with primary antibodies for $12 \mathrm{~h}$ at $4{ }^{\circ} \mathrm{C}$, the membranes were incubated with horseradish peroxidase-conjugated secondary antibodies at room temperature for $1 \mathrm{~h}$ and washed 4-5 times with TBST. The immunocomplexes were visualized using an enhanced chemiluminescence reagent (Bio-Max, Israel).

\section{Statistical analysis}

All data are expressed as the mean \pm standard error from at least 3 independent experiments and were analyzed using SPSS 14.0 software. One-way analysis of variance was used to determine the significance of the differences among the data. Values of $\mathrm{p}<0.05$ were considered statistically significant.

\section{Results}

QLQX alleviated cardiac dysfunction in diabetic mice

We previously found that QLQX has no significant effects on measured cardiac characteristics, including heart rate, left ventricular systolic pressure (LVSP), left ventricular end-diastolic pressure (LVEDP), $\pm \mathrm{dP} / \mathrm{dt}$, and $\mathrm{EF}$, in control mice and rats [9]. Thus, we first 


\section{Cellular Physiology Cell Physiol Biochem 2018;47:1365-1376

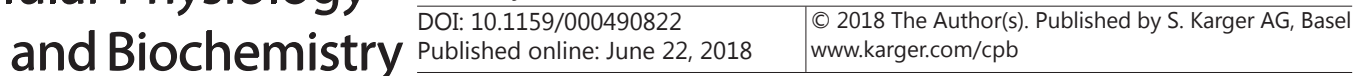 \\ Tong et al.: QLQX Improving Diabetic Cardiac Function}

examined the effects of QLQX in diabetic mice. As expected, after 5 weeks of STZ treatment, the hearts were significantly enlarged (Fig. 1A). However, the thickness of the interventricular septum wall at diastolic phase (IVSd) and the left ventricular posterior wall thickness (LVPWT) did not significantly increase compared with measurements of saline-infused or QLQX-treated mice. Interestingly, the diabetic mice had enlarged left ventricular diameters and decreased IVSd and LVPWT measurements (Table 1 and Fig. 1B). Furthermore, cardiac contractility was significantly decreased in the diabetic mice, as demonstrated by decreased EF and fractional shortening (FS) and increased LVESd. The lung weight to body weight (LW/ $\mathrm{BW}$ ) and heart weight to body weight (HW/BW) ratios were similarly elevated in diabetic mice (Table 1). H\&E-stained histological sections of hearts from the diabetic mice exhibited a damaged phenotype, and using Masson's trichrome staining, we found that cardiac fibrosis was markedly increased in diabetic mice compared with mice administered with saline or QLQX (Fig. 1C-1E). The main reason for this observation is that diabetes caused substantial congestive and systolic cardiac dysfunction, which was significantly attenuated by treatment with QLQX.

Consistent with our previous study [9], glucose levels did not change following the administration of QLQX in diabetic mice; however, weight loss increased significantly, which was possibly related to improved heart function. These results indicated that QLQX did not directly ameliorate diabetes.

Fig. 1. Effects of QLQX on STZinduced histopathological alterations in cardiac tissues. A. Gross morphology of hearts from mice. Diabetic mice exhibited heart enlargement, while QLQX treatment reversed this effect, which was abrogated by TRPV1 deficiency. Additionally, Ang II did not affect STZ-induced heart enlargement in TRPV1-/- mice. B. WT DM and TRPV1 $\%$ DM showed degenerative IVSd and LVPWT, while QLQX treatment reversed this effect in WT DM, but not TRPV1 $\%$ DM. Additionally, Ang II did not change this effect in TRPV1-/- mice (10X). C\&D. Representative Masson's trichrome staining of the ventricles. Increased perivascular (C) and interstitial (D) fibrosis (blue) were observed in WT DM and TRPV1/- DM mice, while QLQX treatment reversed this effect in WT DM mice, but not TRPV1\% DM mice (200X). E. Representativehaematoxylin and eosin (H\&E) staining of the ventricles. Normal architecture of the heart was seen in WT control

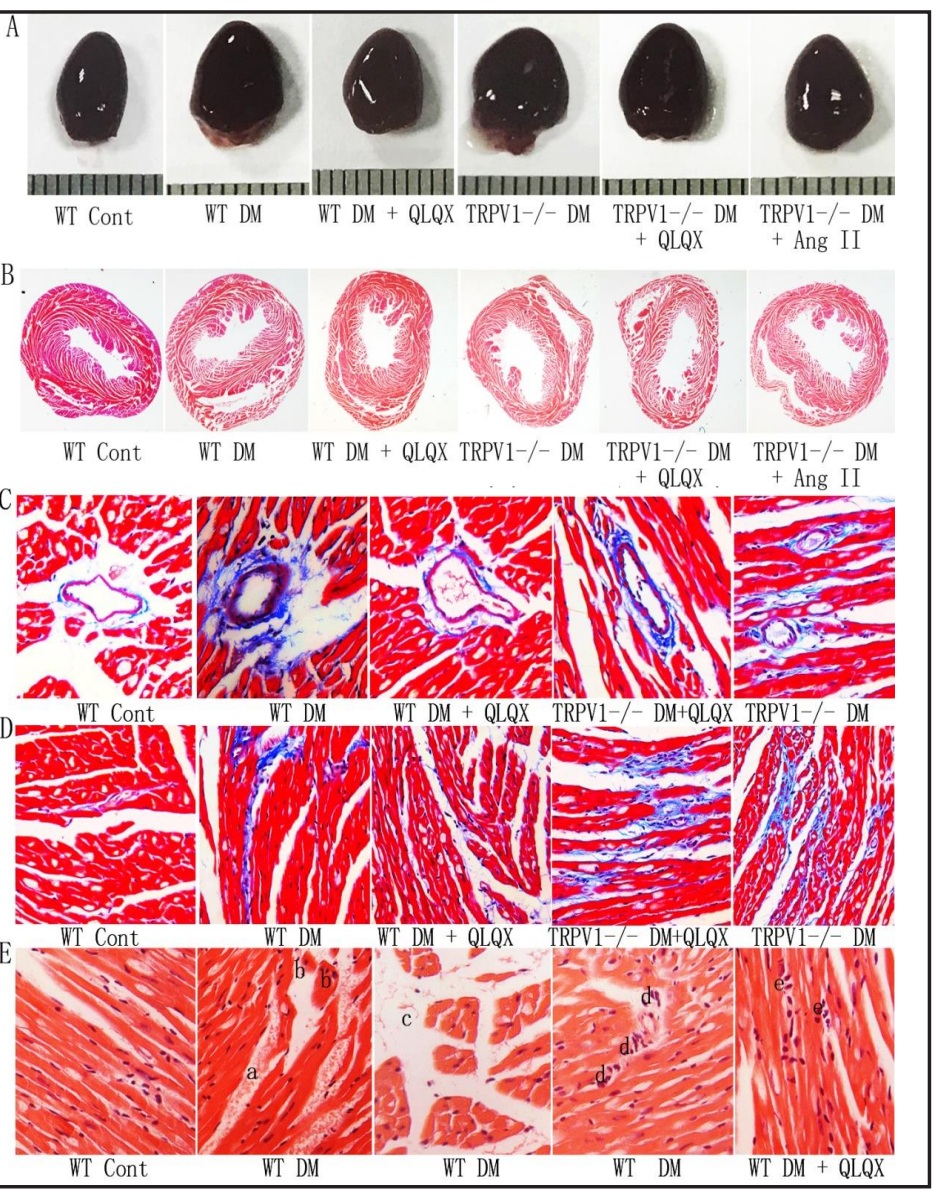
mice. In animals that received STZ, haemorrhage (a), degeneration (b), oedema (c) and fibroblastic proliferation (d) were seen. After treatment with QLQX, only fibroblastic proliferation (e) was seen in diabetic mice. N=5 mice. WT, wild type; Cont, control; DM, diabetes mellitus; QLQX, qiliqiangxin; Ang II, angiotensin II.

\section{KARGER}


AGTR1 participated in the regulation of diabetic cardiac dysfunction following QLQX treatment

We first evaluated AGTR1 expression in diabetic mice. We also examined the expression of ACE1, which mediates the conversion of Ang I to Ang II, after which Ang II couples with AGTR1 to generate adverse effects in cardiac development and the pathogenesis of cardiovascular diseases. As shown in Fig. 2A, diabetes induced high expression of ACE1/AGTR1, while QLQX inhibited diabetes-mediated activation of ACE1/AGTR1. To further assess the effects of AGTR1, we used Ang II or Los, an AGTR1 antagonist, to directly influence the activity of AGTR1 (Fig. 2B). We confirmed that, similar to QLQX, Los effectively decreased the expression of AGTR1, whereas Ang II increased the expression in QLQX-treated diabetic mice. Interestingly, the expression of ACE1 did not change. The effects of Ang II and Los on the development of heart dysfunction were also observed in diabetic mice. Direct inhibition of AGTR1 using pharmacologic therapy (Los) mimicked the improvements in cardiac function mediated by QLQX. Conversely, Ang II almost abolished the beneficial effects of QLQX, including the increased EF and FS and decreased LVESd, HW/BW, and LW/BW (Table 2).

Table 1. Echocardiographic Parameters in Diabetic Mice, body weight, heart weight and glucose in diabetic mice. ${ }^{*} \mathrm{p}<0.05$, compared to WT Control group mice; $\# \mathrm{p}<0.05$, compared to WT DM group mice; $\$ \mathrm{p}<0.05$, compared to WT DM + QLQX group mice; $\mathrm{N}=5$ mice

\begin{tabular}{lllllll} 
Group & \multirow{2}{*}{ WT Cont } & \multirow{2}{*}{ WT DM } & \multirow{2}{*}{ WT DM } & \multirow{2}{*}{ TRPV1/DM+QLQX } & TRPV1/DM+QLQX \\
\cline { 1 - 3 } HR, bpm & $515.3 \pm 12.6$ & $529.7 \pm 15.1$ & $516 \pm 9.58$ & $511.1 \pm 17.8$ & $523 \pm 10.3$ \\
IVSd & $6.77 \pm 1.08$ & $5.0 \pm 0.46^{*}$ & $6.17 \pm 0.71^{\#}$ & $4.93 \pm 0.8^{*}$ & $4.9 \pm 0.62^{\$}$ \\
LVPWT & $7.4 \pm 0.63$ & $5.6 \pm 0.58^{*}$ & $6.67 \pm 0.71^{\#}$ & $5.4 \pm 0.52^{*}$ & $5.37 \pm 0.49^{\$}$ \\
LVEDd & $32.7 \pm 6.73$ & $32.4 \pm 6.66$ & $31.2 \pm 4.98$ & $33.5 \pm 6.5$ & $34.2 \pm 8.25$ \\
LVESd & $20.9 \pm 3.22$ & $23.1 \pm 5.8^{*}$ & $19.8 \pm 3.98^{\#}$ & $24.1 \pm 4.76^{*}$ & $25.3 \pm 4.31^{\$}$ \\
EF\% & $64.9 \pm 10.7$ & $50.9 \pm 6.5^{*}$ & $59.3 \pm 5^{\#}$ & $48.4 \pm 6.8^{*}$ & $49.5 \pm 8.3^{\$}$ \\
FS\% & $37.1 \pm 6.9$ & $28.7 \pm 3.3^{*}$ & $34.5 \pm 4.8^{\#}$ & $27.4 \pm 5.3^{*}$ & $49.5 \pm 8.3^{\$}$ \\
BW, g & $22.9 \pm 3.9$ & $17 \pm 2.6^{*}$ & $20.1 \pm 2.5^{\#}$ & $16.1 \pm 2.5^{*}$ & $16.5 \pm 3.4^{\$}$ \\
HW, mg & $105.7 \pm 10.7$ & $110.7 \pm 11.2$ & $99.7 \pm 9.5$ & $104.3 \pm 8.8$ & $101.3 \pm 9.4$ \\
HW/BW & $4.6 \pm 0.35$ & $6.71 \pm 0.28^{*}$ & $4.98 \pm 0.11^{\#}$ & $6.46 \pm 0.33^{*}$ & $6.26 \pm 0.3^{\$}$ \\
LW/BM & $6.06 \pm 0.28$ & $8.14 \pm 0.31^{*}$ & $6.49 \pm 0.21^{\#}$ & $8.84 \pm 0.33^{*}$ & $6.26 \pm 0.3^{\$}$ \\
Glusoce & $7.95 \pm 1.24$ & $28.49 \pm 4.75^{*}$ & $28.42 \pm 5.82$ & $30.27 \pm 7.63^{*}$ & $29.09 \pm 5.29$ \\
\hline
\end{tabular}

Table 2. Echocardiographic Parameters in Diabetic Mice following Ang II or CL administration. ${ }^{*} \mathrm{p}<0.05$, compared to WT DM group mice; $\# \mathrm{p}<0.05$, compared to WT DM + QLQX group mice; $N=5$ mice

\begin{tabular}{lllll}
\hline Group & \multirow{2}{*}{ WT DM } & $\begin{array}{l}\text { WT DM } \\
\text { QLQX }\end{array}$ & $\begin{array}{l}\text { WT DM } \\
\text { CL }\end{array}$ & $\begin{array}{c}\text { WT DM + QLQX } \\
+ \text { Ang II }\end{array}$ \\
\hline LVEDd, mm & $32.5 \pm 5.27$ & $32.3 \pm 5.17$ & $32.8 \pm 4.72$ & $34.6 \pm 3.75$ \\
LVESd, mm & $24.7 \pm 4.31$ & $21.6 \pm 2.22^{*}$ & $21.9 \pm 3.76^{*}$ & $25.4 \pm 3.85^{\#}$ \\
LVESd, mm & $45.2 \pm 5.8$ & $59.3 \pm 7.8^{*}$ & $57.4 \pm 7.7^{*}$ & $43.6 \pm 2.9^{\#}$ \\
FS\% & $26.7 \pm 5.1$ & $34.9 \pm 4.6^{*}$ & $35.3 \pm 3.3^{*}$ & $26.2 \pm 5.0^{\#}$ \\
HW/BW, & $6.62 \pm 0.43$ & $4.77 \pm 0.58^{*}$ & $4.81 \pm 0.14^{*}$ & $6.85 \pm 0.28^{\#}$ \\
mg/g & & & & \\
LW/BW, & $8.85 \pm 0.44$ & $5.94 \pm 0.23^{*}$ & $6.03 \pm 0.55^{*}$ & $8.6 \pm 0.67^{\#}$ \\
mg/g & & & & \\
\hline
\end{tabular}

Fig. 2. ACE1/AGTR1 expression in diabetic mice. A. DM induced ACE1/AGTR1 expression, whereas QLQX inhibited ACE1/AGTR1 expression. * $\mathrm{p}<0.05$ vs WT Cont; \#p<0.05 vs WT DM. B. Los mimicked the decrease in AGTR1 caused by QLQX, whereas Ang II re-activated AGTR1 in diabetic mice following QLQX administration. ${ }^{*} \mathrm{p}<0.05$ vs WT DM; $\#$ \# $<0.05$ vs WT DM+QLQX. C. TRPV1 KO did not change the DM-induced ACE1/AGTR1 modulation. ${ }^{*} \mathrm{p}<0.05$ vs WT Cont. D. QLQX inhibited ACE1/ AGTR1 expression despite the absence of TRPV1. ${ }^{*} \mathrm{p}<0.05$ vs WT DM; \#p<0.05 vs WT DM+QLQX. E. Ang II increased ACE1/AGTR1 expression in TRPV1 KO mice. * $\mathrm{p}<0.05$ vs TRPV1-/- DM; \#p<0.05 vs TRPV1-/- DMA+QLQX. $\alpha$-Tubulin was used as the internal reference. $\mathrm{N}=5$ mice.

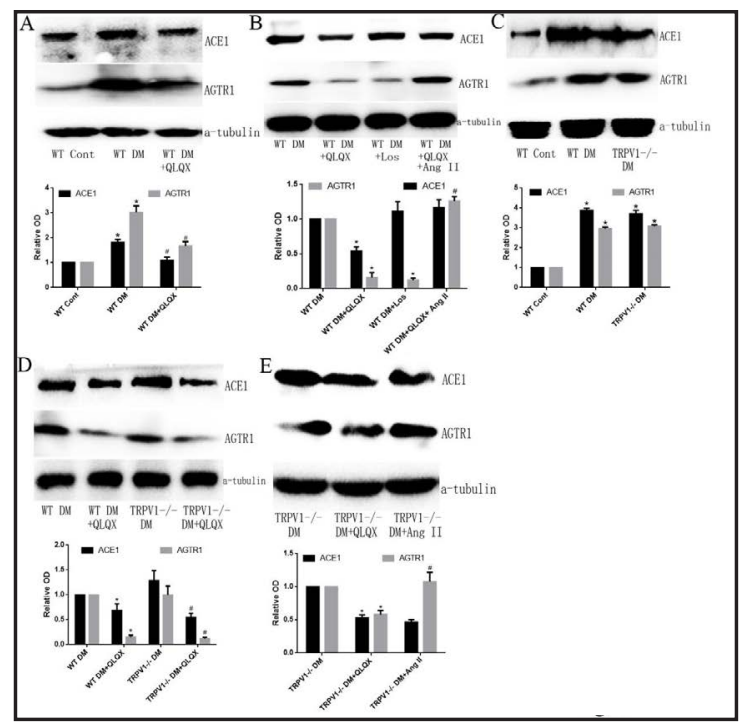




\section{Cellular Physiology Cell Physiol Biochem 2018;47:1365-1376 \begin{tabular}{ll|l} 
and Biochemistry Published online: June 22, 2018 & $\begin{array}{l}\text { C } 2018 \text { The Author(s). Published by S. Karger AG, Basel } \\
\text { www.karger.com/cpb }\end{array}$ \\
\hline
\end{tabular} \\ Tong et al.: QLQX Improving Diabetic Cardiac Function}

TRPV1 appeared to be involved in QLQX-mediated improvement of diabetic cardiac function

As shown in Fig. 3A, TRPV1 expression was decreased in diabetic mice, while after 5 weeks of QLQX administration, the decreased expression of TRPV1 was rescued, indicating that TRPV1 may act as a protective factor in diabetic mice during QLQX treatment. To further elucidate the role of TRPV1, we observed the effects of QLQX on TRPV1 knockout (KO) mice (TRPV1\%) (Fig. 3B) following STZ treatment. As expected, we observed increased LVESd, and HW/BW and LW/BW ratios and decreased EF and FS (Table 1) in QLQX-treated diabetic TRPV1\% mice. Furthermore, histological staining revealed that QLQX attenuated cardiac hemorrhage, degeneration, edema, fibroblastic proliferation, and perivascular and interstitial fibrosis in diabetic mice, whereas the absence of TRPV1 nearly abolished these beneficial effects of QLQX (Fig. 1A-1D). These data indicated that QLQX mediated cardiac protection via the regulation TRPV1 in diabetic mice.

TRPV1 is a potential downstream adaptor of AGTR1 in the diabetic heart

ACE1/AGTR1 was increased while TRPV1 was decreased in the diabetic heart (Fig. 2A, $3 \mathrm{~A}$ ); therefore, we examined the expression of TRPV1 in diabetic hearts following Los or Ang II treatment. As shown in Fig. 3C, after Los treatment, TRPV1 expression was increased, whereas Ang II abrogated the QLQX-mediated increase in TRPV1 expression in diabetic mice. These data indicated that Los and Ang II may function via TRPV1 in diabetic mice. To further elucidate this potential relationship, we examined the effect of TRPV1 KO on ACE1/AGTR1. Interestingly, TRPV1 deficiency did not reverse the activation of ACE1/AGTR1 caused by diabetes (Fig. 2C). Additionally, QLQX still reduced the expression of ACE1/AGTR1 in TRPV1\% diabetic mice compared with WT diabetic mice (Fig. 2D). Finally, although QLQX or Ang II effectively altered the expression of ACE1/AGTR1 in TRPV1\% diabetic mice (Fig. 2E), heart enlargement (Fig. 1A) and IVSd and LVPWT degeneration (Fig. 1B) were not affected by either QLQX or Ang II. Based on these results, we concluded that TRPV1 limited the dysfunctional cardiac responses to AGTR1 in diabetic mice.

\section{QLQX rescued cardiomyocyte autophagy by regulating AGTR1/TRPV1 in diabetic mice}

Previous studies have confirmed that diabetes suppresses cardiac autophagy and induces cardiomyocyte apoptosis, which indicates that autophagy deficiency is important in the pathogenesis of diabetic cardiac dysfunction $[15,16]$. Moreover, it is thought that many AGTR1- or TRPV1-induced effects are mediated by autophagy $[17,18]$. In addition to TRPV1, other biomarkers of autophagy, such as LC3-II, were decreased in diabetic mice (Fig. 4A). Although controversial, p62 accumulation is typically interpreted as a symptom of lysosomal degradation and the exact opposite of enhanced autophagic flux [19]. Consistent with this hypothesis, we observed higher levels of phosphorylated p62 (p-p62) than p62 in diabetic mice (Fig. 4A). Moreover, both the formation of autophagosomes and autolysosomes and the expression of their marker LAMP2 were down-regulated in diabetic mice (Figs. 4A and 5). Additionally, we observed that QLQX induced autophagic flux in diabetic mice. As shown in Figs. 4A and 5, QLQX partially restored the loss of autophagy in diabetic mice. We also found that Los and Ang II affected the QLQX-mediated increase in autophagy (Figs. 4B and

Fig. 3. TRPV1 expression in diabetic mice. A. DM inhibited TRPV1 expression, whereas QLQX increased TRPV1 expression. * ${ }^{\mathrm{p}}<0.05$ vs WT Cont; $\#$ p $<0.05$ vs WT DM. B. We effectively knocked out TRPV1 expression. * ${ }^{*}<0.05$ vs WT Cont. C. QLQX or Los induced TRPV1 expression in diabetic mice, whereas Ang II abrogated TRPV1 activation following QLQX-treatment. ${ }^{*} \mathrm{p}<0.05$ vs WT DM; $\# \mathrm{p}<0.05$ vs WT DM+QLQX. $\alpha$-Tubulin was used as the internal reference. $\mathrm{N}=5$ mice.

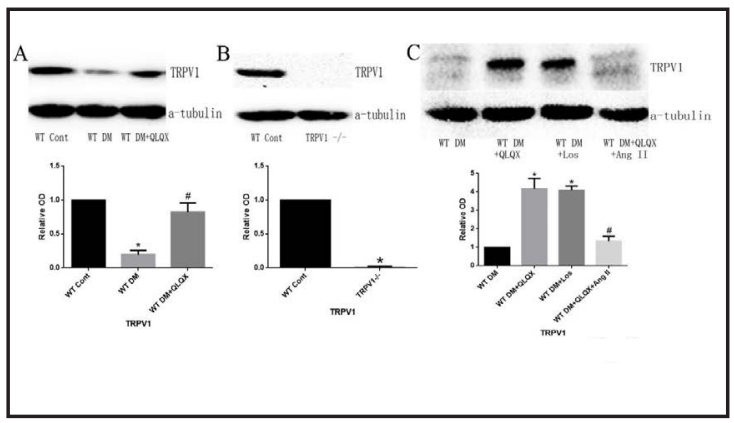




\section{Cellular Physiology Cell Physiol Biochem 2018:47:1365-1376 \begin{tabular}{l|l} 
DOI: 10.1159/000490822 & and Biochemistry \\
Published online: June 22, 2018 & $\begin{array}{l}\text { 2018 The Author(s). Published by S. Karger AG, Basel } \\
\text { www.karger.com/cpb }\end{array}$
\end{tabular} Tong et al.: QLQX Improving Diabetic Cardiac Function}

Fig. 4. QLQX activated autophagic hallmarks expression in diabetic hearts via AGTR1. A. DM induced deficiencies in autophagy, whereas QLQX rescued cardiomyocyte autophagy. ${ }^{*} \mathrm{p}<0.05$ vs WT Cont; \#p<0.05 vs WT DM. B. Los mimicked QLQXmediated autophagy augmentation, whereas Ang II abolished the QLQX-induced increase in autophagy in diabetic mice. ${ }^{*} \mathrm{p}<0.05$ vs WT DM; $\#$ p $<0.05$ vs WT DM+QLQX. N=5 mice.

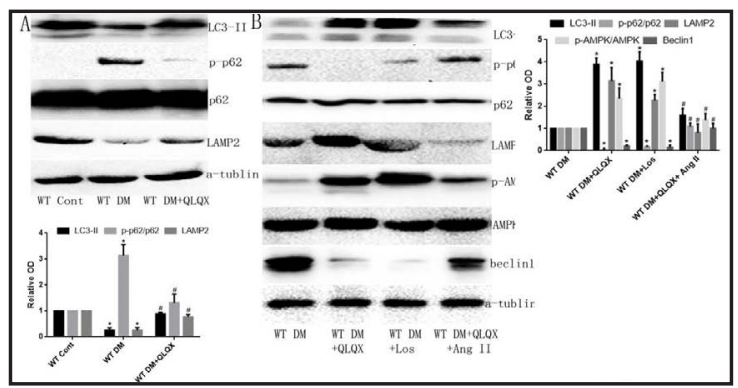

Fig. 5. Representative autophagosome formation in DM hearts. The double membrane structures of autophagic vacuoles were increased in QLQX- or Los-treated WT DM mice, and co-administration of Ang II reduced the number of autophagic vacuoles. Additionally, the number of autophagic vacuoles did not increase in TRPV1\% DM mice following QLQX treatment (original magnification $\times 10000$, higher magnification images $(\times 50000)$ for the selected area show in the respective lower right red insets). $\mathrm{N}=5$ mice.

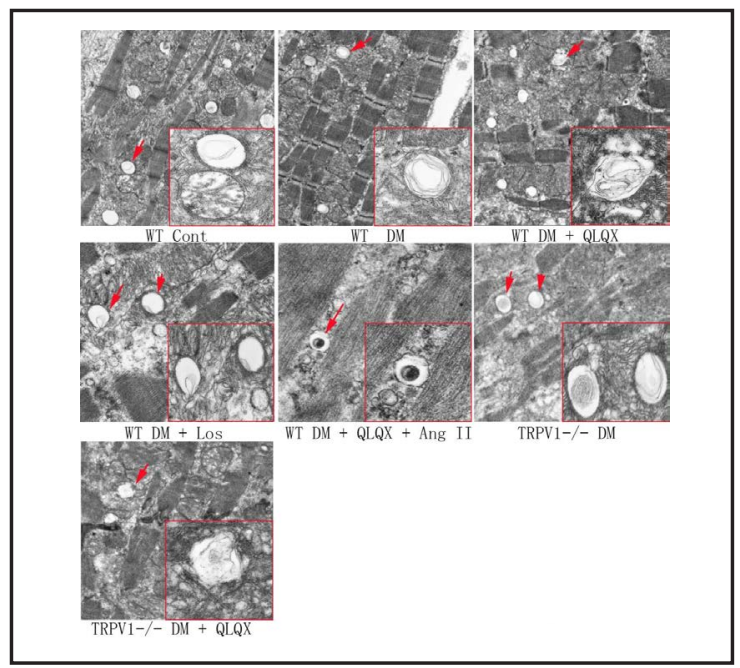

Fig. 6. QLQX increased the expression of autophagic hallmarks in diabetic hearts in the presence of TRPV1, but not in the absence of TRPV1. ${ }^{*} \mathrm{p}<0.05$ vs WT DM; $\# \mathrm{p}<0.05$ vs WT DM+QLQX; $\$ \mathrm{p}<0.05$ vs TRPV1-/- DM. N=5 mice.

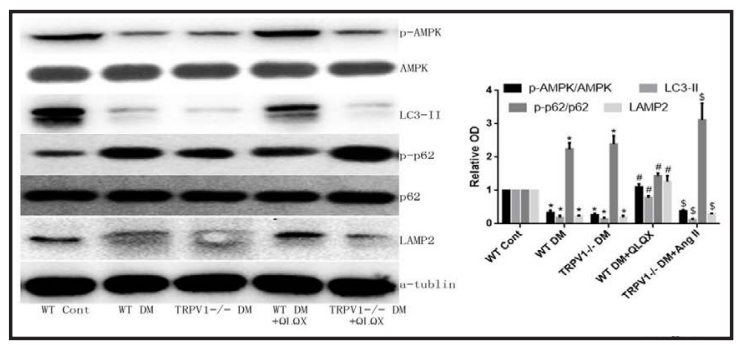

5). Interestingly, p-AMPK/AMPK and beclin1 levels showed a different pattern, indicating that AMPK-dependent and beclin1-dependent autophagy may have adverse effects in diabetic mice following the administration of QLQX. To further investigate the protective effect of TRPV1 on autophagy, autophagy hallmarks and the formation of autolysosomes were examined in TRPV1\% mice. We observed fewer autolysosomes, lower LC3-II and LAMP2 levels, and increased p-p62/p62 expression in diabetic TRPV1\% mice treated with QLQX (Figs. 5 and 6). Based on these data, we concluded that QLQX protected against cardiac damage by regulating AGTR1/TRPV1-mediated autophagy in diabetic mice.

\section{Discussion}

In this study, we showed that QLQX improves autophagy via a TRPV1-dependent mechanism in the diabetic mouse heart. The effects of Los mimicked the QLQX-induced increase in autophagy, whereas Ang II blocked these positive effects. Moreover, the beneficial effects of QLQX were abolished in TRPV1\% mice. Additionally, neither Los nor Ang II affected heart dysfunction or autophagic flux in TRPV1\% diabetic mice. Therefore, QLQX regulated AGTR1/TRPV1-mediated autophagy, and thus prevented cardiac dysfunction in diabetic mice.

\section{KARGER}


QLQX has been found to effectively and safely improve cardiac function and attenuate cardiac remodeling in myocardial infarction and spontaneously hypertensive rat (SHR) models [4-7]. In diabetic models, we found that QLQX can also prevent the disruption of endothelial integrity and the deterioration of cardiac function [9]. In the present diabetic model, we also found that long-term treatment with QLQX induced a significant increase in both EF and FS and a decrease in LVESd and HW/BW. In addition, after 5 weeks of STZ administration, treatment with QLQX resulted in an improved myocardial phenotype, as demonstrated by H\&E and Masson's trichrome staining, that is, lowered LVESd, reduced myocardial hemorrhage, reduced myocardial necrotic or fibrotic areas, decreased fibroblast proliferation, and diminished interstitial edema. These data suggested that QLQX effectively abolishes cardiac dysfunction during hyperglycemic overload. Additionally, QLQX not only resulted in decreased expression of ACE1 and AGTR1 in the diabetic heart but also simulated the protective effects of Los, which agrees with our previous findings in diabetic aortic rings [9]. Thus, QLQX appears to block cardiac remodeling in cardiomyocytes during hyperglycaemic overload.

Renin-angiotensin system (RAS) is a pluripotent toxic factor and a key contributor in cardiovascular remodeling and subsequent cardiovascular dysfunction. It is thought that many QLQX-induced protective effects are mediated by RAS. Zhou [20] found that QLQX seems to reverse ACE1/AGTR1 activation, cardiac hypertrophy, and heart failure in mice following 4 weeks of pressure overload. Moreover, QLQX has been found to down-regulate the expression of ACE1/AGTR1 in STZ-treated rats and protect against heart failure and vascular endothelial dysfunction [9]. In the present study, we also found that QLQX inhibited the expression of ACE1/AGTR1, whereas Los mimicked the beneficial effects of QLQX in diabetic mice. These data confirmed the causal role of QLQX-inhibited ACE1/AGTR1 activation in the diabetic heart. Many signaling transmitters, such as ROS and NO [21], have been found to be involved in ACE1/AGTR1-mediated damage. TRPV1 has recently been shown to have a complex relationship with AGTR1. While some researchers have reported that plasma Ang II is increased in diabetic rats, the expression of TRPV1 in the wall of the left ventricle is not significantly changed [22], and TRPV1 expression in the organum vasculosum laminae terminalis is increased in SHR rats [13], most studies have confirmed that TRPV1 is decreased during ACE1/AGTR1-induced injury or in pressure overload-induced cardiac hypertrophy [23-25]. Treatment with captopril, an inhibitor of ACE1, can induce TRPV1 extravasation in the airways [26]. Moreover, immunofluorescence analysis has shown that Ang II and TRPV1 are co-expressed in cultured neurons [27]. These data demonstrate that TRPV1 plays key roles in the ACE1/AGTR1-mediated effects. The discrepancies among the findings regarding the expression of TRPV1 may be a result of differing ACE1/AGTR1-mediated effects in different models. In this study, increased ACE1/AGTR1 and decreased TRPV1 levels were observed in diabetic mice. Moreover, Los reactivated TRPV1. Furthermore, although both Ang II and Los efficiently altered the levels of AGTR1 in TRPV1 $\%$ diabetic mice, they did not have any effect on cardiac dysfunction. Together, these data indicate that AGTR1 may regulate TRPV1 and cause further cardiac dysfunction in diabetic mice.

Accumulating evidence indicates that TRPV1 plays an essential role in the underlying processes of heart failure, indicating that the modulation of TRPV1 may be a potential target for CHF treatment in diabetes [2, 28]. Indeed, defects of TRPV1 have been associated with the occurrence of heart failure in type 1 and 2 diabetes mellitus due to their effects on ROS production, $\mathrm{Ca}^{2+}$ transients, eNOS activity, adiponectin and leptin signaling control, the modulation of neuronal activity, microcirculatory vasodilation, and the regulation of myocardial blood flow [12, 29-32]. Direct induction of neurotransmitters downstream of TRPV1, such as CGRP and SP, leads to lowered LVEDP and increased LVSP, $\pm \mathrm{dP} / \mathrm{dt}$, and coronary flow in diabetic mice [33]. In this study, we also found decreased expression of TRPV1 and damaged cardiac function in diabetic mice, which was consistent with previous studies. Interestingly, QLQX efficiently increased the expression of TRPV1 in the diabetic heart and improved cardiac function, which suggests that QLQX might affect cardiac function by modulating TRPV1. Therefore, we examined the effects of QLQX in TRPV1 
KO mice. Surprisingly, QLQX did not have any beneficial effects-as observed by both echocardiography and histopathologic assessment-in TRPV1 KO diabetic mice. These data confirmed the pivotal role of TRPV1 in diabetic mice following QLQX treatment. Additionally, we found that QLQX administration ameliorated weight loss in WT diabetic mice, but not in TRPV1\% diabetic mice, which is consistent with a previous study that showed increased weight loss and deterioration of heart failure in TRPV1 KO mice [34].

Autophagy acts as a central regulator of AGTR1/TRPV1-mediated effects [18, 35]. It is initiated by stress through different mechanisms, which causes autophagosomes to fuse with lysosomes to form autolysosomes, resulting in degradation of the cargo. It is therefore a very important process for survival. Recent studies have demonstrated that diabetes induces harmful effects in the heart via autophagy deficiency [15]. We further investigated the involvement of autophagic flux in the diabetic heart following QLQX administration. As expected, diabetes significantly inhibited autophagy, which was re-activated by QLQX administration. We then observed the effects of Ang II/Los on autophagy. Los administration efficiently restored autophagy in diabetic mice. Interestingly, Ang II combined with AGTR1 induces adequate autophagic flux in TAC-mediated cardiac hypertrophy [36]; however, in our study, the inhibition of AGTR1 via Los increased the levels of LC3 and LAMP2 and the number of autophagolysosomes, whereas Ang II abolished these effects. The main reason for this discrepancy is that AGTR1 has different effects in diabetic models. Additionally, we noticed that AMPK-dependent autophagy, which is usually considered beneficial, increased following QLQX or Los treatment, whereas beclin1-dependent autophagy, which is usually considered harmful, increased following Ang II administration in QLQX-treated diabetic mice [37]. These data suggest that the induction of autophagy might be an important mechanism that enables QLQX to regulate the AGTR1 pathway and improve cardiac dysfunction. TRPV1 is an important downstream regulator of AGTR1; therefore, we further investigated whether TRPV1 is involved in the activation of autophagy in QLQX-treated diabetic mice. Recent studies have demonstrated that TRPV1 relies on the AMPK pathway to activate autophagy [34]. During pressure overload-induced cardiac hypertrophy and contractile dysfunction, cold stress accentuates injury through increased expression of TRPV1 receptors, AMPK phosphorylation, and the activation of autophagy [38]. Additionally, AMPK-dependent autophagy has been observed in the TRPV1-mediated inhibition of vascular smooth muscle foam cell formation [17]. Similar pathways for the induction of autophagy by TRPV1 and AMPK have been shown in thymocytes [39]. In our present study, autophagy deficiency and reduced TRPV1 expression were observed in diabetic mice, as indicated by decreased numbers of autophagolysosomes and the expression of LC3II and LAMP2. AMPK phosphorylation was also reduced in diabetic mice. Therefore, we concluded that TRPV1 may play an important role in diabetes-mediated loss of autophagy. We next wondered whether TRPV1 mediates the promotion of autophagy after QLQX administration. First, we found that QLQX increased the expression of TRPV1. QLQX also increased the number of autophagolysosomes, the expression of LC3II and LAMP2 and the phosphorylation of AMPK, and decreased the phosphorylation of $\mathrm{p} 62$ in WT diabetic mice but not in TRPV1\% diabetic mice. These results suggested that QLQX induces autophagy by activating TRPV1 in the diabetic heart.

The limitations of this study should be noted. Although we have shown that TRPV1 is a downstream regulator of ACE1/AGTR1, the effects of Los and Ang II on autophagy in TRPV1 KO diabetic mice were not investigated. Additionally, capsaicin, a direct TRPV1 stimulator, was not used in this study. Further in vivo studies are needed to verify the above mechanisms.

In conclusion, diabetes increased ACE1/AGTR1 activity and decreased autophagy, TRPV1 expression, and cardiac function. QLQX inhibited ACE1/AGTR1 expression, activated autophagy and TRPV1, and improved cardiac dysfunction. Blocking AGTR1 via Los mimicked the beneficial effects mediated by QLQX, whereas Ang II abolished these effects. Moreover, the beneficial effects were observed in WT diabetic mice but not in TRPV1\% diabetic mice. Many questions regarding the molecular network underlying these responses must be answered. However, the possibility that AGTR1/TRPV1-mediated autophagy could play an 


\section{Cellular Physiology Cell Physiol Biochem 2018;47:1365-1376

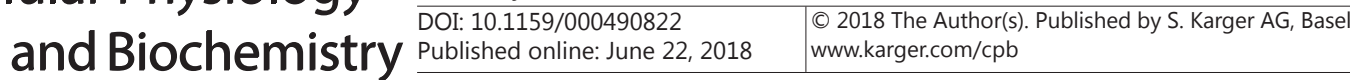 \\ Tong et al.: QLQX Improving Diabetic Cardiac Function}

essential role in QLQX-induced improvements in cardiac dysfunction in diabetic mice is very exciting.

\section{Abbreviations}

HR (heart rate); IVSd (interventricular septum wall thickness at diastolic phase); LVPWT (LV posterior wall thickness; LVEDd); LV (end-diastolic dimension; LVESd,); left (ventricular end-systolic diameter); EF (ejection fraction); FS (fractional shortening); BW (body weight); HW (heart weight); HW/BW (heart weight/body weight); LW/BW (lung weight/body weight).

\section{Acknowledgements}

We thank MSD Corp. for kindly providing Los. We thank Yiling Corp. for kindly providing QLQX. We also thank Prof. Liang-rong Zheng for kindly providing the TRPV1\% mice. Moreover, we thank Prof. Fei Zheng for helping us carry out the western blot experiments. This (work was supported by the China National Natural Science Foundation, Nos: 81670403, 81500381 and 81370390.).

\section{Disclosure Statement}

The authors declare to have no competing interests.

\section{References}

-1 Lozano R, Naghavi M, Foreman K: Global and regional mortality from 235 causes of death for 20 age groups in 1990 and 2010: a systematic analysis for the Global Burden of Disease Study 2010. Lancet 2012;380:2095-2128.

- Derbenev AV, Zsombok A: Potential therapeutic value of TRPV1 and TRPA1 in diabetes mellitus and obesity. Semin Immunopathol 2016;38:397-406.

3 Li X, Zhang J, Huang J, Ma A, Yang J, Li W, Wu Z, Yao C, Zhang Y, Yao W, Zhang B, Gao R: Efficacy and safety of QiliQiangxin capsules for chronic heart failure study group: A multicenter, randomized, double-blind, parallel-group, placebo-controlled study of the effects of qiliqiangxin capsules in patients with chronic heart failure. J Am Coll Cardiol 2013;62:1065-1072.

-4 Tao LC, Shen ST, Fu SY, Fang HY, Wang XZ, Das S, Sluijter JPG, Rosenzweig A, Zhou YL, Kong XQ Xiao JJ, Li XL: Traditional Chinese Medication Qiliqiangxin attenuates cardiac remodeling after acute myocardial infarction in mice. Sci Rep 2015; 5:8374.

5 Wang JF, Zhou JM, Ding XF, Zhu LT, Jiang K, Fu MQ, Wang SJ, Hu K, Ge JB: Qiliqiangxin improves cardiac function and attenuates cardiac remodeling in rats with experimental myocardial infarction. Int J Clin Exp Pathol 2015;8:6596-6606.

6 Wei Y, Liu X, Hou L, Che W, The E, Jhummon MV: Qiliqiangxin affects L type $\mathrm{Ca}^{2+}$ current in the normal and hypertrophied rat heart. Evid Based Complement Alternat Med 2012;2012:131830.

7 Zhou YL,Fang HY, Lin SH, Shen ST, Tao LC, Xiao JJ, Li XL: Qiliqiangxin protects against cardiac ischemiareperfusion injury via activation of the mTOR pathway. Cell Physiol Biochem 2015;37:454-464

-8 Lin SH, Wu XT, Tao LC, Bei YH, Zhang HF, Zhou YL, Shen ST, Xiao JJ, Li XL: The metabolic effects of traditional Chinese medication Qiliqiangxin on H9C2 cardiomyocytes. Cell Physiol Biochem 2015;37:2246-2256.

-9 Chen F, Wu JL, Fu GS, Mou Y, Hu SJ: Chronic treatment with Qiliqiangxin ameliorates aortic endothelial cell dysfunction in diabetic rats. J Cardiovasc Pharmacol Ther 2015; 20:230-240.

10 Katz SD, Hryniewicz K, Hrilajac I, Balidemaj K, Dimayuga C, Hudaihed A, Yasskiy A: Vascular endothelial dysfunction and mortality risk in patients with chronic heart failure. Circulation 2005;111:310-314. 


\section{Cellular Physiology Cell Physiol Biochem 2018;47:1365-1376 \begin{tabular}{l|l} 
DOI: 10.1159/000490822 & O 2018 The Author(s). Published by S. Karger AG, Basel \\
www.karger.com/cpb
\end{tabular} \\ Tong et al.: QLQX Improving Diabetic Cardiac Function}

11 Issa AT, Miyata K, Heng V, Mitchell KD, Derbenev AV: Increased neuronal activity in the OVLT of Cyp1a1Ren2 transgenic rats with inducible Ang II-dependent malignant hypertension. Neurosci Lett 2012;519:2630 .

12 Zheng LR, Han J, Yao L, Sun YL, Jiang DM, Hu SJ, Shao L, Sun ZH, Wang LH: Up-regulation of calcitonin generelated peptide protects streptozotocin-induced diabetic hearts from ischemia/reperfusion injury. Int J Cardiol 2012;156:192-198.

13 DelloStritto DJ, Connell PJ, Dick GM, Fancher IS, Klarich B, Fahmy JN, Kang PT, Chen YR, Damron DS, Thodeti CK, Bratz IN: Differential regulation of TRPV1 channels by H2O2: implications for diabetic microvascular dysfunction. Basic Res Cardiol 2016;111:21.

14 Liu Y, Wang S, Wang C, Song HX, Han HM, Hang PZ, Jiang YN, Wei LL, Huo R, Sun LH, Gao X, Lu YJ, Du ZM: Upregulation of M3 muscarinic receptor inhibits cardiac hypertrophy induced by angiotensin II. J Trans Med 2013;11:209.

15 Zou MH, Xie Z: Regulation of interplay between autophagy and apoptosis in the diabetic heart. Autophagy 2013; 9:624.

16 Yang F, Zhang L, Gao Z, Sun X, Yu M, Dong S, Wu J, Zhao Y, Xu C, Zhang W, Lu F: Exogenous H2S protects against diabetic cardiomyopathy by activating autophagy via the AMPK/mTOR pathway. Cell Physiol Biochem 2017;43:1168-1187.

17 Dai DF, Johnson SC, Villarin JJ, Chin MT, Nieves-Cintrón M, Chen T, Marcinek DJ, Dorn GW 2nd, Kang YJ, Prolla TA, Santana LF, Rabinovitch PS: Mitochondrial oxidative stress mediates angiotensin II-induced cardiac hypertrophy and Gaq overexpression-induced heart failure. Circ Res 2011;108:837-846.

18 Li BH, Yin YW, Liu Y, Pi Y, Guo L, Cao XJ, Gao CY, Zhang LL, Li JC: TRPV1 activation impedes foam cell formation by inducing autophagy in ox-LDL-treated vascular smooth muscle cells. Cell Death Dis 2014; 5:e1182.

19 Patschan S, Chen J, Gealekman O, Krupincza K, Wang M, Shu L, Shayman JA, Goligorsky MS: Mapping mechanisms and charting the time course of premature cell senescence and apoptosis: lysosomal dysfunction and ganglioside accumulation in endothelial cells. Am J Physiol Renal Physiol 2008;294:F100109.

20 Zou Y, Lin L, Ye Y, Wei J, Zhou N, Liang Y, Gong H, Li L, Wu J, Li Y, Jia Z, Wu Y, Zhou J, Ge J: Qiliqiangxin inhibits the development of cardiac hypertrophy, remodeling, and dysfunction during 4 weeks of pressure overload in mice. J Cardiovasc Pharmacol 2012;59:268-280.

21 Sandra D, Erika T, Marina Z, Lucia M: Sulfhydryl angiotensin converting enzyme inhibitor promotes endothelial cell survival through nitric-oxide synthase, fibroblast growth factor-2, and telomerase crosstalk. J Pharmacol Exp Ther 2010;332:776-784.

22 Zhang L, Xiong XQ, Fan ZD, Gan XB, Gao XY, Zhu GQ: Involvement of enhanced cardiac sympathetic afferent reflex in sympathetic activation in early stage of diabetes. J Appl Physiol 2012;113:47-55.

23 Hollis M, Wang DH: Transient receptor potential vanilloid in blood pressure regulation. Curr Opin Nephrol Hypertens 2013;22:170-176.

-24 Wang Q, Ma S, Li D, Zhang Y, Tang B, Qiu C, Yang Y, Yang D: Dietary capsaicin ameliorates pressure overloadinduced cardiac hypertrophy and fibrosis through the transient receptor potential vanilloid type 1. Am J Hypertens 2014;27:1521-1529.

-25 Anand U, Sinisi M, Fox M, MacQuillan A, Quick T, Korchev Y, Bountra C, McCarthy T, Anand P: Mycolactonemediated neurite degeneration and functional effects in cultured human and rat DRG neurons: Mechanisms underlying hypoalgesia in Buruli ulcer. Mol Pain 2016;20:12.

26 de Oliveira JR, Otuki MF, Cabrini DA, Brusco I, Oliveira SM, Ferreira J, André E: Involvement of the TRPV1 receptor in plasma extravasation in airways of rats treated with anangiotensin-converting enzyme inhibitor. Pulm Pharmacol Ther 2016;41:25-33.

27 Anand U, Yiangou Y, Sinisi M, Fox M, MacQuillan A, Quick T, Korchev YE, Bountra C, McCarthy T, Anand P: Mechanisms underlying clinical efficacy of Angiotensin II type 2 receptor (AT2R) antagonist EMA401 in neuropathic pain: clinical tissue and in vitro studies. Mol Pain 2015;11:38.

-28 Aghazadeh Tabrizi M, Baraldi PG, Baraldi S, Gessi S, Merighi S, Borea PA: Medicinal chemistry, pharmacology, and clinical implications of TRPV1 receptor antagonists. Med Res Rev 2017;37:936-983. 


\section{Cellular Physiology Cell Physiol Biochem 2018;47:1365-1376 \begin{tabular}{l|l} 
DOI: 10.1159/000490822 & and Biochemistry 2018 The Author(s). Published by S. Karger AG, Basel \\
wubw.karger.com/cpb
\end{tabular} \\ \begin{tabular}{lll} 
Published online: June 22, 2018 & www.karger.com/cp \\
\hline
\end{tabular}}

29 Khomula EV, Viatchenko-Karpinski VY, Borisyuk AL, Duzhyy DE, Belan PV, Voitenko NV: Specific functioning of Cav3.2 T-type calcium and TRPV1 channels under different types of STZ-diabetic neuropathy. Biochim Biophys Acta 2013;1832:636-649.

-30 Guarini G, Ohanyan VA, Kmetz JG, Dellostritto DJ, Thoppil RJ, Thodeti CK, Meszaros JG, Damron DS, Bratz IN: Disruption of TRPV1-mediated coupling of coronary blood flow to cardiac metabolism in diabetic mice: role of nitric oxide and BK channels. Am J Physiol Heart Circ Physiol 2012;303:H216-23.

-31 Lee Y, Hong S, Cui M, Sharma PK, Lee J, Choi S: Transient receptor potential vanilloid type 1 antagonists: A patent review (2011-2014). Expert OpinTher Pat 2015;25:291-318.

-32 Marche P, Dubosi S, Abraham P, Parot-Schinkel E, Gascoin L, Humeau-Heurtier A, Ducluzeau PH, Mahe G: Neurovascular microcirculatory vasodilation mediated by C-fibers and Transient receptor potential vanilloid-type-1 channels (TRPV1) is impaired in type 1 diabetes. Sci Rep 2017;7:44322.

33 Zheng LR, Zhang YY, Han J, Sun ZW, Zhou SX, Zhao WT, Wang LH: Nerve growth factor rescues diabetic mice heart after ischemia/reperfusion injury via up-regulation of the TRPV1 receptor. J Diabetes Complicat 2015;29:323-328.

-34 Lee E, Jung DY, Kim JH, Patel PR, Hu X, Lee Y, Azuma Y, Wang H-F, Tsitsilianos N, Shafiq U, Kwon JY, Lee HJ, Lee KW, Kim JK: Transient receptor potential vanilloid type-1 channel regulates diet-induced obesity, insulin resistance, and leptin resistance. FASEB J 2015;29:3182-3192.

-35 Maiese K: Warming up to new possibilities with the capsaicin receptor TRPV1: mTOR, AMPK, and erythropoietin. Curr Neurovasc Res 2017;14:1-6.

-36 Porrello ER, D’Amore A, Curl CL, Allen AM, Harrap SB, Thomas WG, Delbridge LM: Angiotensin II type 2 receptor antagonizes angiotensin II type 1 receptor-mediated cardiomyocyte autophagy. Hypertension 2009; 53:1032-1040.

-37 Chen F, Sun ZW, Ye LF, Fu GS, Mou Y, Hu SJ: Lycopene protects against apoptosis in hypoxia/ reoxygenation-induced H9C2 myocardioblast cells through increased autophagy. Mol Med Rep 2015;11:1358-1365.

-38 Lu S, Xu D: Cold stress accentuates pressure overload-induced cardiac hypertrophy and contractile dysfunction: role of TRPV1/ AMPK-mediated autophagy. Biochem Biophys Res Commun 2013;442:8-15.

39 Farfariello V, Amantini C, Santoni G: Transient receptor potential vanilloid 1 activation induces autophagy in thymocytes through ROS-regulated AMPK and Atg4C pathways. J Leukoc Biol 2012;92:421-431. 